\title{
Technical aspects of total aortic repair in the surgical management of acute type A aortic dissection
}

\author{
Sean D. Galvin ${ }^{1}$, Nisal K. Perera ${ }^{2}$, George Matalanis ${ }^{2}$ \\ ${ }^{1}$ Department of Cardiothoracic Surgery, Wellington Regional Hospital, Wellington, New Zealand; ${ }^{2}$ Department of Cardiac Surgery, The Austin \\ Hospital, Heidelberg, Australia \\ Correspondence to: A/Professor George Matalanis. Director, Department of Cardiothoracic Surgery, The Austin Hospital, PO BOX 555, Heidelberg, \\ Victoria 3084, Australia. Email: george.matalanis@austin.org.au.
}

Submitted May 02, 2016. Accepted for publication May 05, 2016.

doi: 10.21037/acs.2016.05.01

View this article at: http://dx.doi.org/10.21037/acs.2016.05.01

Traditional surgical techniques for the management of acute type A aortic dissection (ATAAD) focus on open distal anastomosis with or without hemiarch replacement under a period of deep hypothermic circulatory arrest. This is associated with high rates of false lumen (FL) patency, which exposes the patient to the risk of ongoing end-organ malperfusion and to the formation of complex arch and thoracoabdominal dissection aneurysms. Furthermore, persistent malperfusion is a major source of morbidity and mortality and is not easily reversed following traditional central repair.

Arch replacement using the "branch first" technique allows for complete root, ascending and arch replacement (1-3). A long landing zone is created for proximal endografting with a covered stent. Extended thoracoabdominal stenting is performed in patients with:

(I) Ongoing or recurrent branch vessel ischaemia or malperfusion;

(II) Radiological true lumen (TL) collapse;

(III) Rapid dilatation of the FL;

(IV) Markers of compromised TL perfusion.

The following video (Video 1) illustrates a case of TAAD with clinical and radiological malperfusion and shows the benefits of "branch first" arch replacement followed by stent grafting in this situation.

\section{Clinical vignette}

We present a 74-year-old female with sudden onset of chest pain associated with left leg claudication. Past medical history includes coronary artery bypass grafting in 2001 with a left internal thoracic artery (LITA)-right internal thoracic artery (RITA) "Y-graft" with three distal anastomoses to the left anterior descending, diagonal and obtuse marginal arteries. A right nephrectomy had been performed in 2003 and she had chronic renal failure with an eGFR of $36 \mathrm{~mL} / \mathrm{min}$. She was in chronic atrial fibrillation treated with warfarin and a permanent pacemaker had been placed for symptomatic bradycardia. Other comorbidity included a hemicolectomy, partial thyroidectomy and hypertension. A computed tomography (CT) aortogram confirmed ATAAD with a large FL in the ascending aorta, dissection of the three arch branch vessels, the left renal artery which supplied a solitary left kidney was perfused by the FL and there was occlusion of the left common iliac artery. In addition to the presence of a 'hostile' reoperative sternotomy, dissection of the left subclavian artery threatened the perfusion of the LITARITA "Y-graft". The patient also had chronic renal failure with a solitary left kidney arising from the FL, persistent malperfusion of which is likely to lead to renal failure and dialysis. Finally the patient had symptomatic malperfusion of the left leg with radiological signs of left common iliac artery occlusion.

\section{Operative details}

Bilateral radial and left femoral arterial pressure lines are placed. Cerebral monitoring is by electroencephalogram bispectral index (BIS) and near-infrared spectroscopy (NIRS). The patient is draped widely to allow access to both groins, the neck and the anterior chest. The previous sternotomy 
incision is re-opened down to the sternum and the old wires are removed. In this case the innominate artery has been exposed above the sternal notch. This will allow the rapid establishment of controlled antegrade cerebral perfusion, should cardiac injury be encountered at the re-operative sternotomy.

\section{Cannulation and establishment of cardiopulmonary bypass}

Femoral cannulation is via a direct cutdown and, using a Seldinger technique, a guidewire is placed in both the femoral artery and vein. Transesophageal echocardiography (TEE) confirms the position of each wire, in the right atrium for the venous line and in the TL in the descending thoracic aorta for the arterial line. The cannulas are placed and peripheral femoro-femoral CPB is commenced.

\section{Branch first arch replacement}

Re-operative sternotomy is performed with an oscillating saw. The thymus is divided in the midline and the innominate vein is exposed, mobilized and all tributaries ligated. Each arch branch is exposed for a length of 3-4 cm using a "no-touch" technique. The innominate artery is clamped just proximal to its bifurcation and about one $\mathrm{cm}$ distal to its origin from the arch and transected between the clamps. The distal stump is anastomosed to the first limb of the trifurcation arch graft with a perfusion side arm port (TAPP graft, Vascutek Ltd., Renfrewshire, Scotland, UK). The graft is de-aired and allowed to back-bleed to remove both air and any potential debris from the limbs. The side arm of the TAPP graft is now connected to a separate cerebral head circuit which is used for antegrade flow. The arch reconstruction then proceeds in a similar fashion with reconstruction of the left common carotid artery. Access to the left subclavian in this case is difficult and the left subclavian artery anastomosis is undertaken following depressurisation of the arch. During systemic cooling, the aortic valve is resuspended and the root prepared. Once the desired temperature is reached, the clamp is removed and under a period of moderate systemic circulatory arrest, the distal arch anastomosis is constructed with an anteflow graft (Vascutek Ltd., Renfrewshire, Scotland, UK). Antegrade systemic perfusion is then recommenced via the anteflow limb, and the anteflow graft is connected to the root graft in an end-end fashion. The TAPP graft, which has been passed posterior to the innominate vein, is then connected proximally. Hot shot is administered and the cross clamp removed. Following rewarming and reperfusion, the patient is weaned from bypass, decannulation is performed and haemostasis checked. The soft tissue and pericardium are closed over the prosthetic grafts.

\section{Completion stent grafting}

In this case, there was concern about ongoing branch vessel compromise, and so the patient is transferred directly to the endovascular suite. A covered stent graft (Zenith TX2 TAA Endovascular Graft, Cook Medical, Bloomington, IN, USA) is introduced and positioned within the predesigned dacron proximal landing zone. The remaining thoracic and abdominal aorta is then lined with bare metal uncovered stent grafts (Zenith Dissection endovascular stent, Cook Medical Inc., Bloomington, IN, USA). An angioplasty balloon (Coda Balloon Catheter, Cook Medical Inc., Bloomington, IN, USA) is then used to sequentially expand the bare metal stents and rupture the septum between the TL and FL to create a single aortic channel. This patient also required stent grafting to the left renal artery. At the conclusion of the procedure, aortography is performed and the patency of the aortic lumen and the visceral and other branch vessels are confirmed. The patient remains well at follow up, now three years after the dissection. Follow up CT scan shows a single aortic lumen with patency of all branch vessels.

\section{Comments}

The "branch first" arch technique has become our standard technique for surgical management of ATAAD. The technique of total aortic repair described above allows the successful management of ATTAD with reliable FL obliteration of all dissected thoracoabdominal aorta. In doing so branch vessel ischemia is treated, TL perfusion is ensured, and aortic healing is encouraged while the aorta is provided structural support from the stent graft.

\section{Acknowledgements}

None.

\section{Footnote}

Conflicts of Interest: The authors have no conflicts of interest to declare. 


\section{References}

1. Galvin SD, Matalanis G. Continuous perfusion "Branchfirst" aortic arch replacement: a technical perspective. Ann Cardiothorac Surg 2013;2:229-34.

2. Matalanis G, Galvin SD. "Branch-first" continuous perfusion aortic arch replacement and its role in intra-

Cite this article as: Galvin SD, Perera NK, Matalanis G. Technical aspects of total aortic repair in the surgical management of acute type A aortic dissection. Ann Cardiothorac Surg 2016;5(3):248-250. doi: 10.21037/acs.2016.05.01 operative cerebral protection. Ann Cardiothorac Surg 2013;2:194-201.

3. Matalanis G, Perera NK, Galvin SD. Aortic arch replacement without circulatory arrest or deep hypothermia: the "branch-first" technique. J Thorac Cardiovasc Surg 2015;149:S76-82. 